\title{
Universal Data Logger System for Environmental Monitoring Applications
}

\author{
Osman Abd Allah ${ }^{1 \star}$, Mohammed Abdalla ${ }^{2}$, Suliman Abdalla ${ }^{3}$, Amin Babiker ${ }^{4}$, and Alaa \\ Awad Allah ${ }^{5}$ \\ ${ }^{1,2,5}$ Sudan Academy of Science, Khartoum, Sudan \\ ${ }^{3}$ Sudan Atomic Energy Commission, Khartoum, Sudan \\ ${ }^{4}$ Faculty of Engineering, Alneelain University, Khartoum, Sudan \\ e-mail: abudahdah@live.com
}

\begin{abstract}
Collecting huge amount of data in long time acquisition systems like in environmental monitoring, there is a need to collect and save data over time for further use or analysis. A data logger is an electronic device that records data over time or in a relation to location either with a built-in instrument, sensor or via an external instruments and sensor. In this paper, a data logger system is designed to use as a standalone or computer based device. When used as a standalone system, all data acquired are saved in SD memory card, which must be copied and erased periodically depending on the memory size. When used as computer based device, all the data sent to the computer via the serial port and stored automatically in achieved files. The limit of those files size only restricted by the capacity of the disk. The data logger is designed using an Arduino UNO board and LabView software, and it has the flexibility to set it up for different user options. With this system, the user could be able to record and read back sensory data to or from existing files, or in automatically generated files and plot these readings in a graph. Also, the user have the ability to choose the periodic time at which a sample record in a file in term of seconds, minuits or hours. The system designed to monitor and record a single channel data, but it could be adapted to monitor more than one channel.
\end{abstract}

Keywords: Data Logger, Monitoring, SD memory, Arduino, LabView

\section{Introduction}

A data logger is an electronic instrument that records environmental parameters such as temperature, relative humidity, wind speed and direction, light intensity, water level and water quality over time[1,2]. Typically, data loggers are compact, battery-powered devices that are equipped with microprocessor input channels and data storage. Most data loggers utilize turnkey software on a personal computer to initiate the logger and view the collected data. The use of data loggers for environmental monitoring became common during the 1980's; coinciding with the explosion in personal computers (PC's), since a data logger system consists of many of the same, or similar, components used to manufacture a PC. Before then, chart recorders were commonly used as well as manual measurements. Both of these methods were labor intensive and time consuming so the advent of stand-alone data loggers was welcomed. [3]

A data logger used to collect readings, or output, from sensors. These sensors could be measuring industrial parameters such as pressure, flow and temperature or environmental parameters such as water level, wind speed or solar radiation. Today there are sensors available, which can virtually measure any physical parameter. Sensors developed to measure gas pressure within human cells to cloud height and density[4].

Data loggers in general needs eight basic components to achieve a proper function[5]:

a. Input channel: The output from a sensor connected to a data logger channel. A channel consists of circuitry designed to 'channel' a sensor signal (typically a voltage or current) from the sensor to the data logger processor. A single data logger can have a variety of channel types and from one to many channels (multi-channel data logger) - one channel is required for every sensor signal output.

b. Analog to Digital Converter: All sensor signals must be in binary format for the data logger system to recognize and record. Since most of the sensors output is analog, they must be digitized using an ADC before feeding them to the system. 
c. Microprocessor: A processor is the logic circuitry that responds to and processes the basic instructions that drive a computer or data logger.

d. Memory: for storing the data

e. Power supply to power up the data logger system

f. Data output port: Most data loggers communicate with a PC via a serial port, which allows data to be transmitted in a series (one after the other). The RS-232 interface has been a standard for decades as an electrical interface between data terminal equipment, such as a PC, and data communications equipment employing serial binary data interchange, such as a data logger or modem.

g. Whether proof enclosure: Since environmental data loggers are usually installed in remote and harsh locations, a weatherproof case is an obvious requirement. Most loggers are packaged in weather resistant plastic cases.

h. Software: Proprietary software is usually required to program and download data from a data logger. Data logging functions such as sensor scan rate and scaling, log interval, communication protocol and output format (Excel, ASCII, plot, etc.) are programmed using software loaded on a PC.

Most often, data logger systems are portable stand-alone devices, which allocated to measure and record sensory data, then connected to a PC to analyze data. In this work, the system could be used as a stand-alone -as in normal cases- or use any general PC with the software installed to act as a data logger system.

\section{Materials and Method}

The designed data logger system consists mainly from Arduino UNO board as the main controller and data acquisition system. The system represents a single channel that record the temperature over time, which acquired from an LM35 temperature sensor. The system equipped with an SD memory card adaptor, which loaded with a micro SD card for storing files in standalone mode. Figure 1 below shows the system components used in the design.
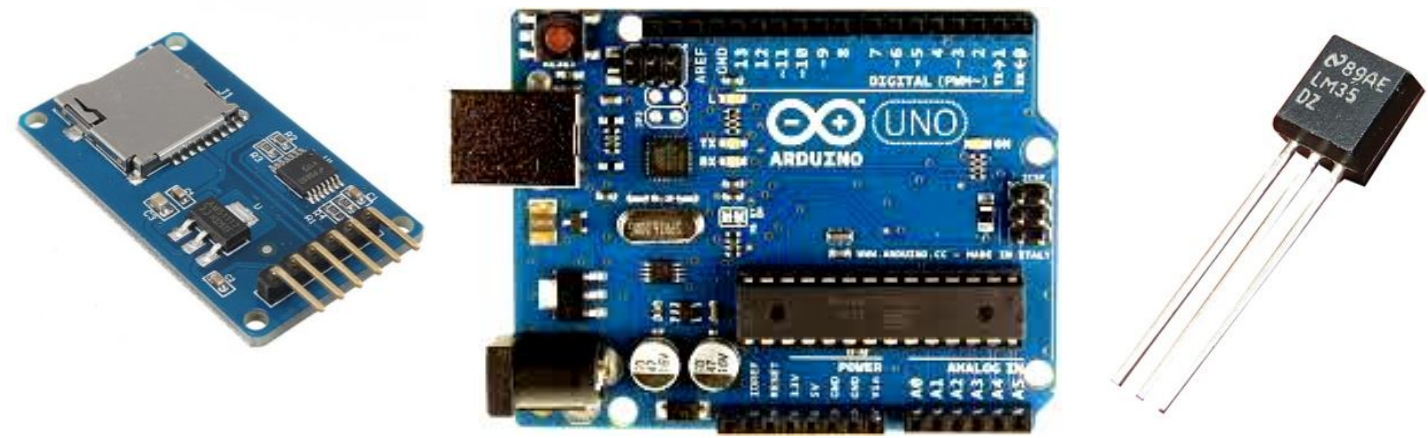

Figure 1. the system components

The adaptor connected to the board and exchange data through the standard SPI interface, and it has the "CS" pin which determined according to the adaptor type or brand and in this case its "8"[6]. In this mode, the card has to be formatted first in FAT/16 or FAT/32 system before using it with the board. The SDFormatter is a suitable program used to format the card [7]. Then the card tested and initialized from the Arduino software and a text file is created in the memory card to store data. Of course, there could be many files in the memory card to save data, but only one file could be opened and if another file is targeted, the first one must be closed before opening the new one. The size of the memory eliminates the files size, and the acquisition time determine the duration at which the memory came to an overflow point. For these reasons, the memory must be copied and cleared periodically.

In the computer-based mode, the board send the data to the PC through the serial port and the system designed using the LabView software from National Instruments, which offers huge graphical programming capabilities makes data logging more flexible and powerful.

IJEEI Vol. 5, No. 2, June 2017: $131-136$ 
LabView block diagram enables dealing with files operations like saving, clearing or even creating files from the program, as well as monitoring and plotting data in a single user interface. The system has the ability to save data in an existing text file, or in a file that created from the program automatically. It also has the option to clear or read any saved file manually through control buttons.

The system could be developed by adding many features such as printing a specific file, determine a critical reading, searching for specific measures in a specific period of time, compare file contents with another, sending data to e-mail or even plotting files data online using loT applications.

\section{The System}

The temperature sensor LM35 connected to the board in the analog pin A0 to read ambient temperature and many other sensors could be added to the design in the same way as desired. In stand-alone mode, the card module connected to the SPI interface, which consists of the standard signals MOSI, MISO, SCK and additional chip select pin (CS) which determined by the type and brand of the adaptor. The board itself connected to the PC through a USB connector used to program the board and send data to the LabView software.

The computer-based system consists of many user-defined options described below.

The read/clear option enables the user to read any file and display its contents in an indicator. It also enables the user to clear any existing file to start over saving.

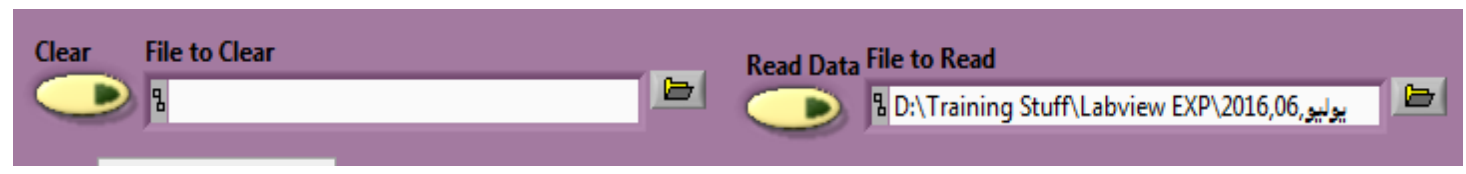

Figure 2. File read/clear options

Saving files option offers two options for saving data, either saving data in a an existing file which called "One File" option, or saving data daily in separate files in the option " Daily". In the first option, the user creates a text file in any directory in the PC and choose that file for saving. In the second option, the system automatically creates a file and name it with the current date, when a new day exists, a different file generated with the new day name.

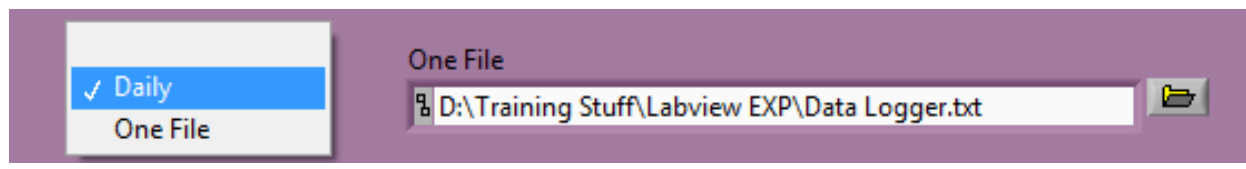

Figure 3. Saving files options

Interval option enables the user to choose an interval scale for saving data either in seconds, minuits or hours and an interval period to determine the specific period for saving data. For example, if the user choose "minuits" in the "interval scale" control and "5" in the "interval period" control, the system will save a sample data every five minuits.

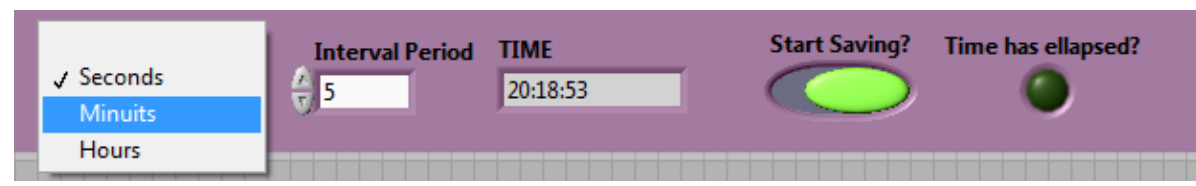

Figure 4. Sample saving intervals options 
In addition, there is an indicator for the local time and a control for start saving data. The LED indicator blinks once when the predefined period for saving data has elapsed.

Monitoring option shows a live reading for the temperature and a graph for plotting the readings instantly.

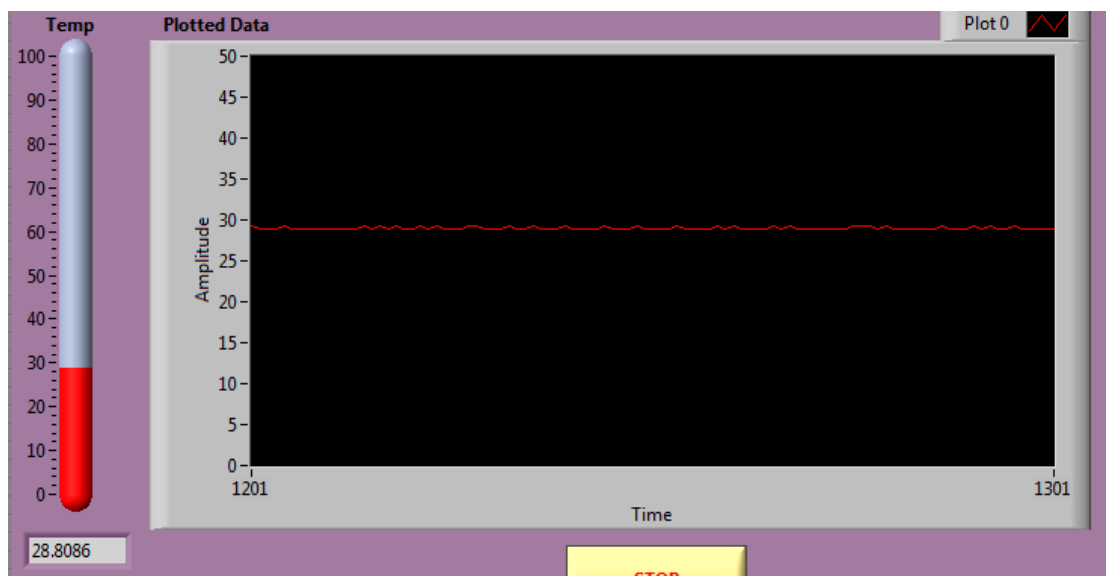

Figure 5. The temperature reading and data plotting

Figure 6 shows the designed block diagram in LabView program, and figure 6 shows the circuit.

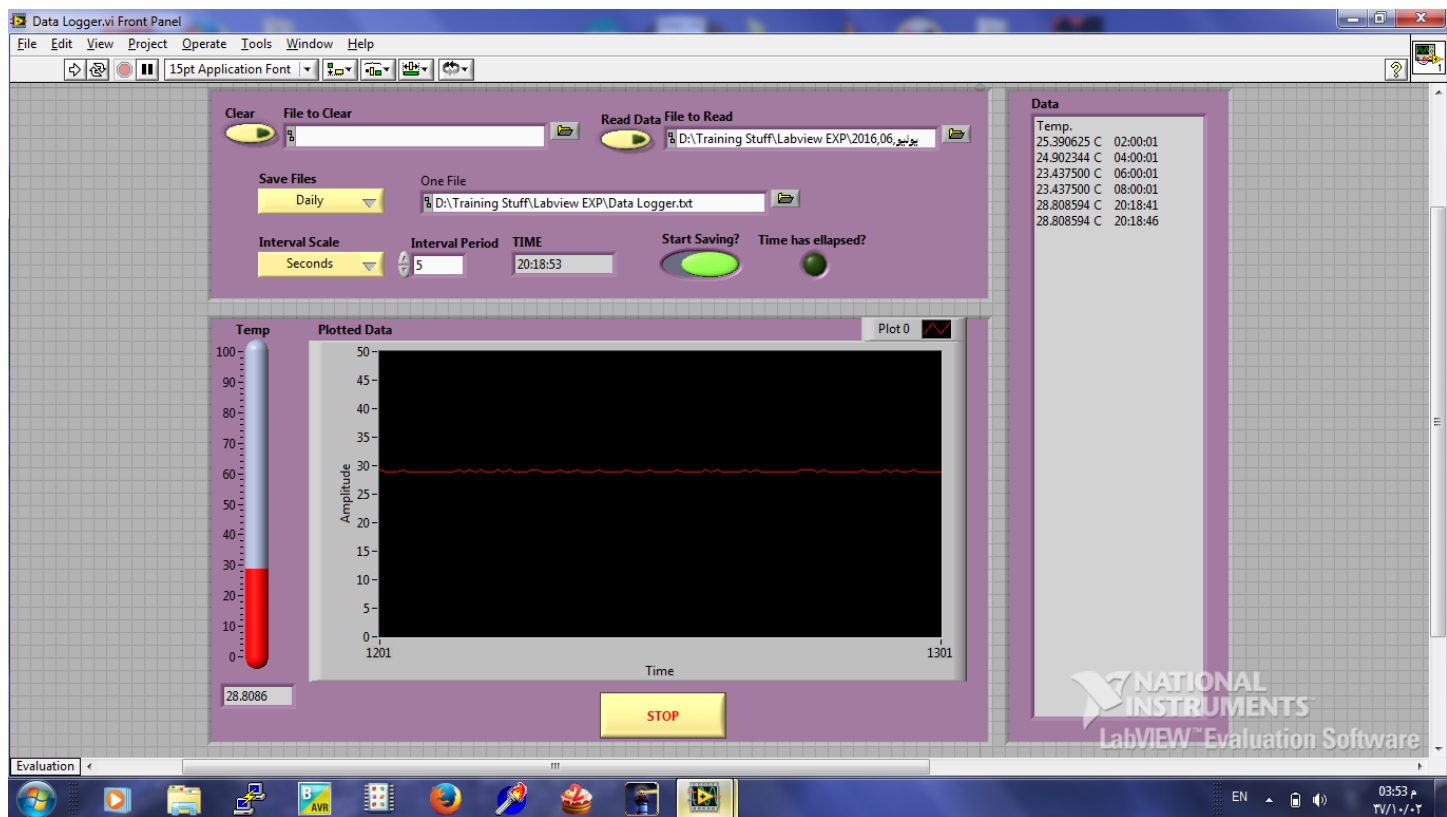

Figure 6. The Data logger system in LabView 


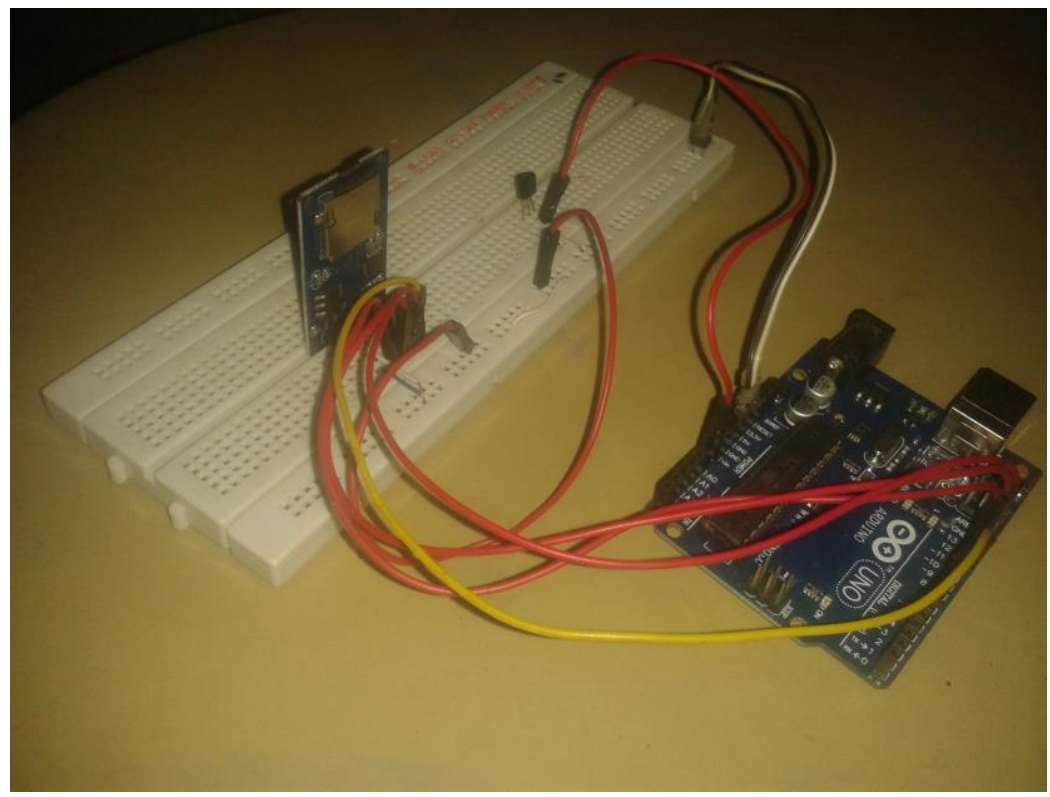

Figure 7. the Data Logger circuit

The figure $8 \mathrm{a}$ and $8 \mathrm{~b}$ shows the files that used to store data while measuring temperature.

\begin{tabular}{|c|c|}
\hline File Edit Format & View Help \\
\hline $\begin{array}{l}\text { Temp. } \\
27.343750 \mathrm{C} \\
26.855469 \mathrm{C} \\
26.855469 \mathrm{C} \\
26.855469 \mathrm{C} \\
26.855469 \mathrm{C} \\
25.878906 \mathrm{C} \\
25.878906 \mathrm{C} \\
26.367187 \mathrm{C} \\
25.878906 \mathrm{C} \\
25.878906 \mathrm{C} \\
25.878906 \mathrm{C} \\
26.367187 \mathrm{C} \\
26.855469 \mathrm{C} \\
26.367187 \mathrm{C} \\
26.367187 \mathrm{C} \\
26.855469 \mathrm{C} \\
27.343750 \mathrm{C} \\
26.855469 \mathrm{C} \\
27.343750 \mathrm{C} \\
26.855469 \mathrm{C} \\
27.343750 \mathrm{C} \\
26.367187 \mathrm{C} \\
27.832031 \mathrm{C} \\
27.832031 \mathrm{C} \\
27.832031 \mathrm{C} \\
27.832031 \mathrm{C} \\
27.832031 \mathrm{C} \\
27.832031 \mathrm{C} \\
27.832031 \mathrm{C} \\
27.343750 \mathrm{C} \\
27.832031 \mathrm{C} \\
27\end{array}$ & $\begin{array}{l}22: 00: 01 \\
22: 05: 01 \\
22: 20: 01 \\
22: 25: 01 \\
22: 30: 01 \\
22: 35: 01 \\
22: 40: 01 \\
22: 45: 01 \\
22: 55: 01 \\
23: 00: 01 \\
23: 05: 01 \\
23: 10: 01 \\
23: 15: 01 \\
23: 20: 01 \\
23: 25: 01 \\
23: 30: 01 \\
23: 35: 01 \\
23: 40: 01 \\
23: 45: 01 \\
23: 50: 01 \\
23: 55: 01 \\
00: 00: 01 \\
18: 11: 31 \\
18: 11: 36 \\
18: 11: 41 \\
18: 11: 46 \\
18: 11: 51 \\
18: 11: 56 \\
18: 12: 01 \\
18: 12: 06 \\
18: 12: 11 \\
18: 12: 16\end{array}$ \\
\hline
\end{tabular}

Figure 8a. Saving data in "One File" option 


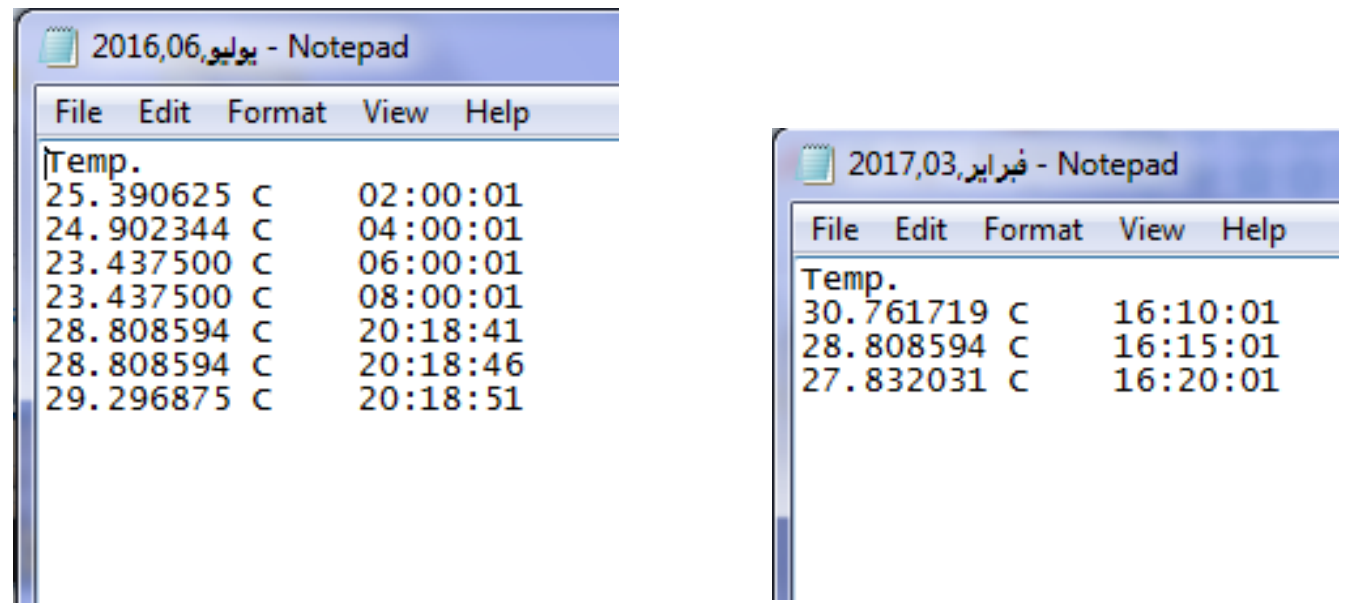

Figure 8b. Saving data in "Daily" option

Figure 8a shows the data stored in a file named as "Data Logger" which is an existing file used to store data in the "One File" option. From the time column, it is clear that the periodic time chosen by the user to save samples is "five Minuits". This option tested and data acquired and saved in different time during the day, which the system can plotted and analyzed.

Figure $8 \mathrm{~b}$ shows two files generated automatically by the system in the "Daily" option, and the file name takes the current date when the data saved during this day. In the daily option, the system automatically generates a new file daily with the appropriate date. The figure shows a file generated in the day 6, July2017 with "Tow Hours" interval for saving data, and the other shows a file generated in the date 3, February 2017 with "Five Minuits" intervals.

\section{Conclusion}

As a result, for testing and evaluating the system, the data logger shows a very good results and it could be developed to adapt any user requirements. The system flexibility enables easy upgrade for multi-channel data logger system for monitoring more environmental changes like humidity, air pressure, wind speed or the percentage of $\mathrm{CO} 2$ gas. Additional features could be also added to the system concerned the files such as print a hard copy via a printer or send a file to a particular e-mail.

\section{References}

[1] Geo scientific Itd. Watershed management instrumentation

[2] Kang J, Park S. Integrated comfort sensing system on indoor climate. Sensors and Actuators. 2000: 302-307.

[3] Ravishanker A, Pandian R. Embedded system based sensor failure detection and industrial environment control over wireless network. 2014.

[4] Riva Marco, Piergiovanni, Schiraldi, Luciano Schiraldi, Alberto. Performances of time-temperature indicators in the study of temperature exposure of packaged fresh foods. Packaging Technology and Science. 2001.

[5] GS Nhivekar, RR Mudholker. Data Logger and Remote Monitoring System for Multiple Parameter Measurement Application, submitted to e -Journal of Science \& Technology (e-JST) (2011)

[6] Information on http://www. www.campbellsci.com

[7] SanDisk Corporation. SanDisk SD card product manual. Version-2.2.2004. www.sandisk.com. 